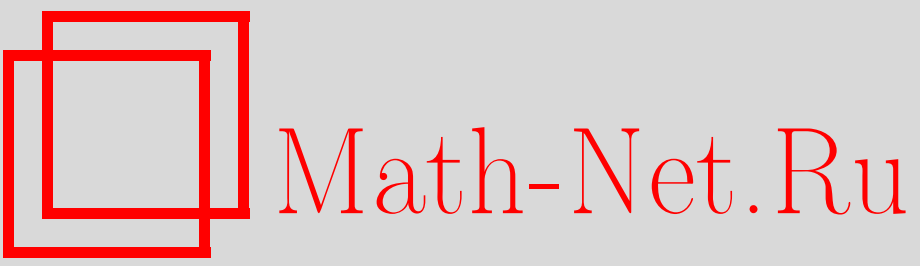

В. А. Смирнов, Э. Р. Рахметов, Стратегия областей в асимптотическом разложении двухпетлевых вершинных фейнмановских диаграмм, ТМФ, 1999, том 120, номер 1, 64-71

DOI: https://doi.org/10.4213/tmf760

Использование Общероссийского математического портала Math-Net.Ru подразумевает, что вы прочитали и согласны с пользовательским соглашением

http://www. mathnet.ru/rus/agreement

Параметры загрузки :

IP : 18.234 .156 .22

26 апреля 2023 г., 18:35:08 
ТЕОРЕТИЧЕСКАЯ

И МАТЕМАТИЧЕСКАЯ

ФИЗИКА

Том 120, № 1

июль, 1999

(C) 1999 г.

В.А. Смирнов* , Э.Р. Рахметов*

\section{СТРАТЕГИЯ ОБЛАСТЕЙ В АСИМПТОТИЧЕСКОМ РАЗЛОЖЕНИИ ДВУХПЕТЛЕВЫХ ВЕРШИННЫХ ФЕЙНМАНОВСКИХ ДИАГРАММ}

\footnotetext{
Обсуждаются и иллюстрируются на двухпетлевых примерах общие правила вычисления коэффициентов при произвольных степенях и логарифмах в асимптотическом разложении фейнмановских диаграмм в судаковском пределе. Описаны особенности, связанные с вычислением отдельных членов в разложении, в частности введение вспомогательной аналитической регуляризации.
}

1. Наиболее простые явные формулы ([1-3], а также [4]) для асимптотических разложений фейнмановских диаграмм в различных пределах импульсов и масс вне массовой оболочки, когда импульсы считаются большими или малыми в евклидовом смысле, были обобщены на некоторые пределы на массовой оболочке, типичные для пространства Минковского [5-7], в частности на судаковский предел. Предписания для этих пределов были сформулированы с использованием (пред)вычитаний в некотором семействе подграфов данного графа.

Недавно были предложены явные предписания для разложения фейнмановских интегралов вблизи порога [8]. Это было сделано с помощью стандартной физической стратегии, основанной на анализе областей в пространстве петлевых импульсов. Следует, однако, отметить, что эта стратегия областей обычно применялась только для вычисления и суммирования ведуших логарифммов, в частности в судаковском пределе (см., например, [9]). Заметим, что информация о ведущих логарифмах присутствует только во вкладах некотрых специфических областей, так что интегрирование по другим областям вообще не рассматривается.

В работе [8] утверждалось (и было продемонстрировано для порогового разложения), что имеет смысл использовать эту стратегию областей для вычисления коэффициентов при любой степени и логарифме в произвольном пределе. В такой расширенной формулировке стратегия сводится к следующим предписаниям:

а) рассмотрим все области петлевых импульсов, типичные для данного предела, и разложим в каждой области подынтегральное выражение в ряд Тейлора по параметрам, которые считаются малыми в данной области;

* Московский государственный университет, Москва, Россия. E-mail: smirnov@theory.npi.msu.su; rahmetov@theory.npi.msu.su. 


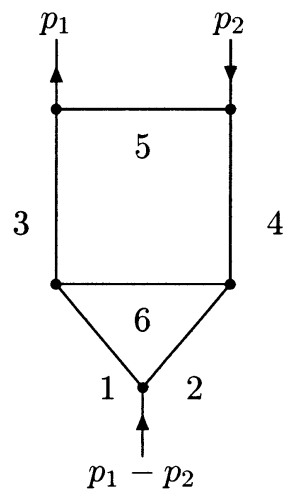

Рис. 1. Двухпетлевая планарная вершинная диаграмма

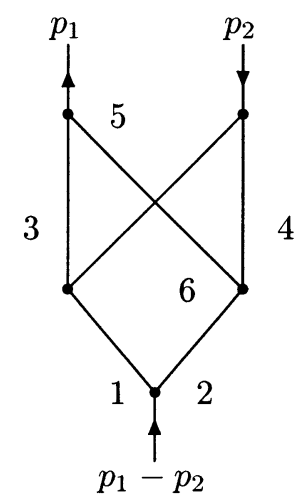

Рис. 2. Двухпетлевая непланарная вершинная диаграмма

б) разложенное (в каждой области по-разному) подынтегральное выражение проинтегрируем по всей области петлевых импульсов;

в) положим равным нулю любой безмасштабный интеграл (даже если он не регуляризован, например, размерной регуляризацией).

Как отмечалось в работе [8], именно шаг "б" далеко не тривиален. Можно верить, что эта стратегия законна для любого предела импульсов и масс. Например, она приводит к хорошо известным формулам для асимптотических разложений в случае типично евклидовых пределов $[1,2]$ (доказанных в [3]), так что мы имеем косвенное подтверждение, по крайней мере для таких теорий. Отметим, что для этих пределов, а также для предела на массовой оболочке, рассмотренного в работах $[5,6]$, набор соответствующих областей определяется разбиением всех петлевых импульсов на большие (жесткие) и малые (мягкие).

В настоящей работе мы проверяем на двухпетлевых примерах эту эвристическую процедуру для вычисления коэффициентов при любой степени и логарифме в асимптотическом разложении фейнмановских диаграмм в судаковском пределе [10]. Мы рассмотрим два общепринятых варианта этого предела для вершинных диаграмм с внешними импульсами $p_{1}, p_{2}$ и $q=p_{1}-p_{2}$.

ПреДЕЛ 1. Два внешних импульса вне массовой оболочки, $p_{1}^{2}=p_{2}^{2}=m^{2}=-\mu^{2}$, $Q^{2} \equiv-q^{2} \rightarrow \infty$; все внутренние массы нулевые.

ПреДЕл 2. Два внешних импульса на массовой оболочке, $p_{1}^{2}=p_{2}^{2}=0, Q^{2} \rightarrow \infty$; некоторые внутренние массы ненулевые.

Мы вычислим ведушее степенное поведение, включая все логарифмы $\ln ^{j}\left(q^{2} / m^{2}\right), j=$ $0,1,2,3,4$, безмассовой планарной диаграммы (рис. 1 ) в первом пределе и сравним полученный результат с известным явным выражением [11]. После такого подтверждения мы применим сформулированное вьше эвристическое предписание к непланарной диаграмме (рис. 2), для которой аналитические результаты неизвестны, в пределе 2 при $m_{1}=\cdots=m_{4}=0, m_{5}=m_{6}=m$. Мы также используем второй пример, для того 
чтобы описать технику вычисления отдельных членов разложения. Естественный способ вычислить члены с зависимостью вида $1 /\left(m^{2}\right)^{2 \epsilon}$ - это введение вспомогательной аналитической регуляризации. В отличие от планарной диаграммы во втором пределе, где возникают полюсы первого порядка по параметру аналитической регуляризации, которые сокращаются в сумме двух вкладов [7], для непланарной диаграммы мы встретим полюсы второго порядка, которые присутствуют в пяти вкладах. Эти полюсы в сумме также сокрашаются, и мы получаем результат, совпадающий с размерной регуляризацией.

2. Фейнмановский интеграл для диаграммы на рис. 1 имеет вид

$$
\begin{aligned}
F_{1}(Q, m, \epsilon)= & \iint \frac{d^{d} k d^{d} l}{\left(l^{2}-2 p_{1} l+m^{2}\right)\left(l^{2}-2 p_{2} l+m^{2}\right)} \times \\
& \times \frac{1}{\left(k^{2}-2 p_{1} k+m^{2}\right)\left(k^{2}-2 p_{2} k+m^{2}\right) k^{2}(k-l)^{2}} .
\end{aligned}
$$

Используется размерная регуляризация [12] с $d=4-2 \epsilon$. При изложении наших результатов мы будем опускать множители $i \pi^{d / 2}$, приходящиеся на каждую петлю, а при выписывании отдельных вкладов в виде разложения по $\epsilon$ мы также опустим множители $\exp \left(-\gamma_{E} \epsilon\right)$, приходящиеся на каждую петлю $\left(\gamma_{E}-\right.$ постоянная Эйлера).

Выберем для удобства внешние импульсы следующим образом:

$$
\begin{array}{ll}
p_{1}=\tilde{p}_{1}+\frac{m^{2}}{Q^{2}} \tilde{p}_{2}, & p_{2}=\tilde{p}_{2}+\frac{m^{2}}{Q^{2}} \tilde{p}_{1}, \\
\tilde{p}_{1}=\left(\frac{Q}{2},-\frac{Q}{2}, 0,0\right), & \tilde{p}_{2}=\left(\frac{Q}{2}, \frac{Q}{2}, 0,0\right),
\end{array}
$$

так что $p_{i}^{2}=m^{2}, \tilde{p}_{i}^{2}=0,2 \tilde{p}_{1} \tilde{p}_{2}=2 \tilde{p}_{1} p_{2}=Q^{2}$. В данном пределе следующие области оказываются типичными [9]:

$$
\begin{aligned}
\text { жсесткая (h): } & k \sim Q, \\
\text { 1-коллинеарная (1c): } & k_{+} \sim Q, \quad k_{-} \sim \frac{m^{2}}{Q}, \quad \underline{k} \sim m, \\
2 \text { 2-коллинеарная }(2 \mathrm{c}): & k_{-} \sim Q, \quad k_{+} \sim \frac{m^{2}}{Q}, \quad \underline{k} \sim m, \\
\text { сверхмягкая (us) : } & k \sim \frac{m^{2}}{Q} .
\end{aligned}
$$

Здесь $k_{ \pm}=k_{0} \pm k_{1}, \underline{k}=\left(k_{2}, k_{3}\right)$. Выражение $k \sim Q$ и т.д. подразумевает, что каждая компонента $k_{\mu}$ имеет порядок $Q$.

Следует считать, что каждый из импульсов $k, l, \ldots$ принадлежит к одному из перечисленных типов, и предусмотреть разные выборы типов петлевых импульсов, избегая двойного счета. Другие области дают нулевые вклады, в частности, когда один из петлевых импульсов является мягким, т.е. при $k \sim m$. Однако, если некоторые массы диаграммы были бы ненулевыми, то некоторые мягкие области породили бы ненулевые вклады (которые бы начинались со следуюшего после ведушего порядка). 
В ведушем порядке $\left(1 / Q^{4}\right)$ мы получаем вклады от следуюших девяти областей: (h-h), (1c-h), (2c-h), (1c-1c), (2c-2c), (us-h), (us-1c), (us-2c), (us-us). В этом списке области для петлевых импульсов $k$ и $l$ в (1) указаны соответственно на первом и втором местах. Область $(\mathrm{h}-\mathrm{h})$ порождает тейлоровское разложение подынтегрального выражения по параметру $m$. В ведушем порядке это не что иное, как значение безмассовой планарной диаграммы при $p_{1}^{2}=p_{2}^{2}=0$, впервые вычисленное в работе [13]. Хотя этот результат можно выразить в гамма-функциях при произвольном $\epsilon$ с помощью метода интегрирования по частям [14] (это было впервые сделано в работе [15]), мы его здесь представляем для краткости в разложении по $\epsilon$ :

$$
\begin{aligned}
C_{(\mathrm{h}-\mathrm{h})}^{(1)} & =\iint \frac{d^{d} k d^{d} l}{\left(l^{2}-2 \tilde{p}_{1} l\right)\left(l^{2}-2 \tilde{p}_{2} l\right)\left(k^{2}-2 \tilde{p}_{1} k\right)\left(k^{2}-2 \tilde{p}_{2} k\right) k^{2}(k-l)^{2}}= \\
& =\left(\frac{1}{4 \epsilon^{4}}+\frac{5 \pi^{2}}{24 \epsilon^{2}}+\frac{29 \zeta(3)}{6 \epsilon}+\frac{3 \pi^{4}}{32}\right) \frac{1}{\left(Q^{2}\right)^{2+2 \epsilon}} .
\end{aligned}
$$

Все вклады, связанные со сверхмягкими областями, легко вычисляются в гамма-функциях с помошью $\alpha$-параметров. В ведушем порядке мы имеем

$$
\begin{aligned}
C_{(\text {us-us })}^{(1)}= & \iint \frac{d^{d} k d^{d} l}{\left(-2 \tilde{p}_{1} l+m^{2}\right)\left(-2 \tilde{p}_{2} l+m^{2}\right)\left(-2 \tilde{p}_{1} k+m^{2}\right)\left(-2 \tilde{p}_{2} k+m^{2}\right) k^{2}(k-l)^{2}}= \\
= & \frac{\Gamma(1-\epsilon)^{2} \Gamma(2 \epsilon)^{2}}{\epsilon^{2}\left(-m^{2}\right)^{4 \epsilon}\left(Q^{2}\right)^{2-2 \epsilon}}, \\
C_{(\text {us-h })}^{(1)}= & \iint \frac{d^{d} k d^{d} l}{\left(l^{2}-2 \tilde{p}_{1} l\right)\left(l^{2}-2 \tilde{p}_{2} l\right)\left(-2 \tilde{p}_{1} k+m^{2}\right)\left(-2 \tilde{p}_{2} k+m^{2}\right) k^{2} l^{2}}= \\
= & \frac{\Gamma(1+\epsilon) \Gamma(1-\epsilon) \Gamma(\epsilon)^{2} \Gamma(-\epsilon)^{2}}{\Gamma(1-2 \epsilon)\left(-m^{2}\right)^{2 \epsilon}\left(Q^{2}\right)^{2}} \\
C_{(\text {us-1c) }}^{(1)}= & \iint \frac{d^{d} k d^{d} l}{\left(-2 \tilde{p}_{1} l\right)\left(l^{2}-2 p_{2} l+m^{2}\right)\left(-2 \tilde{p}_{1} k+m^{2}\right)\left(-2 \tilde{p}_{2} k+m^{2}\right)} \times \\
& \times \frac{1}{k^{2}\left(l^{2}-\frac{\left(2 \tilde{p}_{1} l\right)\left(2 \tilde{p}_{2} k\right)}{Q^{2}}\right)}=\frac{\Gamma(1-\epsilon)^{2} \Gamma(\epsilon) \Gamma(2 \epsilon) \Gamma(-\epsilon)}{\epsilon \Gamma(1-2 \epsilon)\left(-m^{2}\right)^{3 \epsilon}\left(Q^{2}\right)^{2-\epsilon}} \equiv C_{(\text {us-2c) }}^{(1)} .
\end{aligned}
$$

С использованием $\alpha$-параметров остальные вклады можно при произвольном $\epsilon$ представить через интегралы Меллина-Барнса:

$$
\begin{aligned}
C_{(1 \mathrm{c}-1 \mathrm{c})}^{(1)}= & \iint \frac{d^{d} k d^{d} l}{\left(-2 \tilde{p}_{1} l\right)\left(l^{2}-2 p_{2} l+m^{2}\right)\left(-2 \tilde{p}_{1} k\right)\left(k^{2}-2 p_{2} k+m^{2}\right) k^{2}(k-l)^{2}}= \\
= & \frac{\Gamma(\epsilon) \Gamma(-\epsilon) \Gamma(2 \epsilon)}{\Gamma(1+\epsilon)\left(-m^{2}\right)^{2 \epsilon}\left(Q^{2}\right)^{2}} \times \\
& \times \frac{1}{2 \pi} \int_{-i \infty}^{+i \infty} d s \frac{\Gamma(s-3 \epsilon) \Gamma(s+1-2 \epsilon) \Gamma(s+1-\epsilon) \Gamma(\epsilon-s) \Gamma(-s)}{\Gamma(s+1-3 \epsilon)} \equiv \\
\equiv & C_{(2 \mathrm{c}-2 \mathrm{c})}^{(1)},
\end{aligned}
$$




$$
\begin{aligned}
C_{(1 \mathrm{c}-\mathrm{h})}^{(1)}= & \iint \frac{d^{d} k d^{d} l}{\left(l^{2}-2 \tilde{p}_{1} l\right)\left(l^{2}-2 \tilde{p}_{2} l\right)\left(-2 \tilde{p}_{1} k\right)\left(k^{2}-2 p_{2} k+m^{2}\right)} \times \\
& \times \frac{1}{k^{2}\left(l^{2}-\frac{\left(2 \tilde{p}_{1} k\right)\left(2 \tilde{p}_{2} l\right)}{Q^{2}}\right)}=\frac{\Gamma(\epsilon) \Gamma(-\epsilon) \Gamma(1-\epsilon)}{\Gamma(1-2 \epsilon)\left(-m^{2}\right)^{\epsilon}\left(Q^{2}\right)^{2+\epsilon}} \times \\
& \times \frac{1}{2 \pi} \int_{-i \infty}^{+i \infty} d s \frac{\Gamma(s+1) \Gamma(s-\epsilon) \Gamma(s+1+\epsilon) \Gamma(-\epsilon-s) \Gamma(-s)}{\Gamma(s+1-2 \epsilon)} \equiv \\
\equiv & C_{(2 \mathrm{c}-\mathrm{h})}^{(1)} .
\end{aligned}
$$

Мы подразумеваем стандартный способ выбора контуров: ультрафиолетовые (УФ-) полюсы находятся справа, а инфракрасные (ИК-) полюсы - слева от них. Приведенные выше интегралы Меллина-Барнса разлагаются по $\epsilon$ с помощью сдвига контуров и выделения вычетов в точках, где УФ- и ИК-полюсы совпадают при $\epsilon \rightarrow 0$. В результате мы получаем

$$
\begin{gathered}
\left(Q^{2}\right)^{2}\left[C_{(1 \mathrm{c}-1 \mathrm{c})}^{(1)}+C_{(2 \mathrm{c}-2 \mathrm{c})}^{(1)}+C_{(1 \mathrm{c}-\mathrm{h})}^{(1)}+C_{(2 \mathrm{c}-\mathrm{h})}^{(1)}\right]=-\frac{1}{2 \epsilon^{4}}+\left(L^{2}-\frac{\pi^{2}}{2}\right) \frac{1}{2 \epsilon^{2}}+ \\
+\left(\frac{1}{2} L^{3}-\frac{\pi^{2}}{6} L-\frac{17 \zeta(3)}{3}\right) \frac{1}{\epsilon}+\frac{7}{24} L^{4}-4 \zeta(3) L-\frac{\pi^{4}}{144}
\end{gathered}
$$

где $L=\ln \left(Q^{2} / \mu^{2}\right)$ и мы положили для краткости $\mu=1$. (Отметим, что в отдельных вкладах имеются как члены с $\ln \left(Q^{2} / \mu^{2}\right)$, так и с $\ln \left(\mu^{2}\right)$.)

Собирая все девять вкладов вместе, мы наблюдаем, что полюсы по $\epsilon$, которые оказываются очень разной природы (УФ, ИК и коллинеарные), сокращаются, и мы получаем

$$
\left(Q^{2}\right)^{2} F_{1}(Q, m, 0) \stackrel{Q \rightarrow \infty}{\sim} \frac{1}{4} L^{4}+\frac{\pi^{2}}{2} L^{2}+\frac{7 \pi^{4}}{60}
$$

в согласии с разложением хорошо известного результата [11] в ведушем порядке.

3. Разложение планарной диаграммы (см. рис. 1) при $m_{1}=\cdots=m_{4}=0, m_{5}=$ $m_{6}=m$ в пределе 2 было в произвольном порядке получено в работе [7] с помощью стратегии вычитаюших операторов. Отметим, что те же самые выражения для всех вкладов разложения можно получить с помошью стратегии областей. На этом языке список ненулевых вкладов состоит из (h-h), (1c-h), (2c-h), (1c-1c) и (2c-2c), а также вклада, которьй начинается со следующего за ведущим порядка и происходит от области, где импульс центральной линии мягкий, а второй петлевой импульс жесткий.

Рассмотрим теперь разложение непланарной диаграммы (см. рис. 2) в пределе 2. Фейнмановский интеграл можно записать как

$$
\begin{aligned}
F_{2}(Q, m, \epsilon)= & \iint \frac{d^{d} k d^{d} l}{\left((k+l)^{2}-2 p_{1}(k+l)\right)\left((k+l)^{2}-2 p_{2}(k+l)\right)} \times \\
& \times \frac{1}{\left(k^{2}-2 p_{1} k\right)\left(l^{2}-2 p_{2} l\right)\left(k^{2}-m^{2}\right)\left(l^{2}-m^{2}\right)},
\end{aligned}
$$


где $p_{1}$ и $p_{2}$ удовлетворяют соотношениям для $\tilde{p}_{1,2}$ в предыдущем пункте. Мы также используем второй выбор петлевых импульсов, когда $k$ и $l$ являются соответственно импульсами линий 3 и 4 и который получается перестановкой масс и соответствует интегралу (12) с $m_{1}=m_{2}=m_{5}=m_{6}=0, m_{3}=m_{4}=m$.

Ненулевые вклады в разложение в ведущем порядке порождаются следующими областями: (h-h), (h-2c), (2c-h), (1c-1c), (2c-2c), (2c-1c), (1c-1c)', (2c-2c)' и (us-us)'. Как и выше, мы указываем область для петлевого импульса $k$ на первом месте, а для $l$ - на втором. Штрихом обозначены области для второго естественного выбора петлевых импульсов. Вклад (h-h) дается безмассовой непланарной диаграммой. В виде разложения по $\epsilon$ результат можно найти в работе [13]:

$$
C_{(\mathrm{h}-\mathrm{h})}^{(2)}=\left(\frac{1}{\epsilon^{4}}-\frac{\pi^{2}}{\epsilon^{2}}-\frac{83 \zeta(3)}{3 \epsilon}-\frac{59 \pi^{4}}{120}\right) \frac{1}{\left(Q^{2}\right)^{2+2 \epsilon}} .
$$

Вклад (us-us)' легко вычисляется в гамма-функциях:

$$
\begin{aligned}
C_{\left(\text {us-us }^{\prime}\right.}^{(2)} & =\iint \frac{d^{d} k d^{d} l}{\left(-2 p_{1}(k+l)\right)\left(-2 p_{2}(k+l)\right)\left(-2 p_{1} k+m^{2}\right)\left(-2 p_{2} l+m^{2}\right) k^{2} l^{2}}= \\
& =\frac{1}{\left(Q^{2}\right)^{2-2 \epsilon}\left(m^{2}\right)^{4 \epsilon}}[\Gamma(\epsilon) \Gamma(2 \epsilon) \Gamma(1-2 \epsilon)]^{2} .
\end{aligned}
$$

Вклад (2c-h) задается как

$$
\begin{aligned}
C_{(2 \mathrm{c}-\mathrm{h})}^{(2)}= & \iint \frac{d^{d} k d^{d} l}{\left(l^{2}-2 p_{1} l+\frac{\left(2 p_{2} k\right)\left(2 p_{1} l\right)}{Q^{2}}\right)\left(l^{2}-2 p_{2}(k+l)+\frac{\left(2 p_{2} k\right)\left(2 p_{1} l\right)}{Q^{2}}\right)} \times \\
& \times \frac{1}{\left(k^{2}-2 p_{1} k\right)\left(l^{2}-2 p_{2} l\right)\left(k^{2}-m^{2}\right) l^{2}},
\end{aligned}
$$

а совпадаюший с ним (h-2c)-вклад ведушего порядка получается перестановкой $k$ и $l$. Используя $\alpha$-параметры и (дважды) представление Меллина-Барнса, мы получаем

$$
C_{(\mathrm{h}-2 \mathrm{c})}^{(2)}=C_{(2 \mathrm{c}-\mathrm{h})}^{(2)}=\left(-\frac{3}{\epsilon^{4}}+\frac{\pi^{2}}{\epsilon^{2}}+\frac{22 \zeta(3)}{\epsilon}+\frac{16 \pi^{4}}{45}\right) \frac{1}{\left(Q^{2}\right)^{2+\epsilon}\left(m^{2}\right)^{\epsilon}} .
$$

Вклад (1c-1c) имеет вид

$$
\begin{aligned}
C_{(1 \mathrm{c}-1 \mathrm{c})}^{(2)}= & \iint \frac{d^{d} k d^{d} l}{\left(-2 p_{1}(k+l)\right)\left((k+l)^{2}-2 p_{2}(k+l)\right)} \times \\
& \times \frac{1}{\left(-2 p_{1} k\right)\left(l^{2}-2 p_{2} l\right)\left(k^{2}-m^{2}\right)\left(l^{2}-m^{2}\right)},
\end{aligned}
$$

а вклад (2c-2c) получается перестановкой $k$ и $l$. Мы должны также рассмотреть аналогичные вклады $(1 \mathrm{c}-1 \mathrm{c})^{\prime}$ и $(2 \mathrm{c}-2 \mathrm{c})^{\prime}$ со вторым выбором петлевых импульсов. Соответствующие выражения получаются перестановкой масс (см. выше). Пятый ненулевой вклад коллинеарно-коллинеарного типа происходит из области (2c-1c). Оказывается, 
что эти вклады регуляризованы размерно только в сумме. Удобно ввести вспомогательную аналитическую регуляризацию в линии 3 и 4 , подставив

$$
\frac{1}{\left(k^{2}-2 p_{1} k\right)^{1+x_{1}}\left(l^{2}-2 p_{2} l\right)^{1+x_{2}}}
$$

в подынтегральное выражение. В отличие от планарной двухпетлевой диаграммы в этом пределе [5] у нас появляются полюсы по $x_{i}$ вплоть до второго порядка. В частности, вклад (2c-1c) вычисляется в гамма-функциях при призвольном $\epsilon$ :

$$
\begin{aligned}
C_{(2 \mathrm{c}-1 \mathrm{c})}^{(1)}= & \iint \frac{d^{d} k d^{d} l}{\left(-2 p_{1} l+\frac{\left(2 p_{2} k\right)\left(2 p_{1} l\right)}{Q^{2}}\right)\left(-2 p_{2} k+\frac{\left(2 p_{2} k\right)\left(2 p_{1} l\right)}{Q^{2}}\right)} \times \\
& \times \frac{1}{\left(k^{2}-2 p_{1} k\right)\left(l^{2}-2 p_{2} l\right)\left(k^{2}-m^{2}\right)\left(l^{2}-m^{2}\right)}= \\
= & \frac{\Gamma\left(x_{1}\right) \Gamma\left(x_{2}\right) \Gamma\left(-x_{1}-\epsilon\right) \Gamma\left(-x_{2}-\epsilon\right) \Gamma\left(x_{1}+\epsilon\right) \Gamma\left(x_{2}+\epsilon\right)}{\Gamma\left(1+x_{1}\right) \Gamma\left(1+x_{2}\right) \Gamma(-\epsilon)^{2}\left(-m^{2}\right)^{x_{1}+x_{2}+2 \epsilon}\left(Q^{2}\right)^{2}} .
\end{aligned}
$$

С использованием техники $\alpha$-параметров и представления Меллина-Барнса для других (е-е)-вкладов мы получаем результат в виде разложения по $x_{i}$. Затем мы выключаем аналитическую регуляризацию (сначала $x_{2} \rightarrow x_{1}$, а затем $x_{1} \rightarrow 0$ ), замечаем, что в сумме всех пяти вкладов выпадает сингулярная зависимость по $x_{i}$, и получаем следуюший результат в разложении по $\epsilon$ :

$$
\begin{aligned}
&\left(Q^{2}\right)^{2}[\left.C_{(1 \mathrm{c}-1 \mathrm{c})}^{(2)}+C_{(2 \mathrm{c}-2 \mathrm{c})}^{(2)}+C_{(1 \mathrm{c}-1 \mathrm{c})^{\prime}}^{(2)}+C_{(2 \mathrm{c}-2 \mathrm{c})^{\prime}}^{(2)}+C_{(2 \mathrm{c}-1 \mathrm{c})}^{(2)}\right]= \\
&= \frac{19}{4 \epsilon^{4}}-\frac{9}{2 \epsilon^{3}} L+\left(L^{2}-\frac{11 \pi^{2}}{4}\right) \frac{1}{2 \epsilon^{2}}-\left(\frac{3 \pi^{2}}{4} L+\frac{97 \zeta(3)}{6}\right) \frac{1}{\epsilon}+ \\
& \quad+\frac{\pi^{2}}{12} L^{2}+9 \zeta(3) L-\frac{23 \pi^{4}}{32}
\end{aligned}
$$

где $L=\ln \left(Q^{2} / m^{2}\right)$ и мы положили для краткости $m=1$.

Собирая все вклады ведушего порядка, видим, что полюсы по $\epsilon$ сокращаются, и мы приходим к следующему результату:

$$
\left(Q^{2}\right)^{2} F_{2}(Q, m, 0) \stackrel{Q \rightarrow \infty}{\sim} \frac{7}{12} L^{4}-\frac{\pi^{2}}{2} L^{2}+20 \zeta(3) L-\frac{31 \pi^{4}}{180} .
$$

С помощью некоторой компюютерной алгебры можно обобщить этот результат на любой порядок разложения по $1 / Q^{2}$.

Благодарности. В. Смирнов благодарен М. Бенеке, А. И. Давыдычеву и К. Г. Четыркину за полезные обсуждения. Работа поддержана Российским фондом фундаментальных исследований (грант № 98-02-16981), а работа В. Смирнова, кроме того, Фольксвагенским фондом (грант № I/73611). 


\section{Список литературы}

[1] S. G. Gorishnii. On the construction of operator expansions and effective theories in the MS-scheme. General formalism. Препринт ОИЯИ Е2-86-176. Дубна: ОИЯИ, 1986; On the construction of operator expansions and effective theories in the MS-scheme. Examples. Infrared finiteness of coefficient functions. Препринт ОИЯИ Е2-86-177. Дубна: ОИЯИ, 1986; Nucl. Phys. B. 1989. V. 319. P. 633.

[2] К.Г. Четьркин. ТМФ. 1988. Т. 75. С. 26; Т. 76. С. 207.

[3] V. A. Smirnov. Commun. Math. Phys. 1990. V. 134. P. 109; Renormalization and asymptotic expansions. Basel: Birkhäuser, 1991.

[4] V. A. Smirnov. Mod. Phys. Lett. A. 1995. V. 10. P. 1485; Asymptotic expansions of Feynman integrals on the mass shell in momenta and masses. In: Proc. of the 5th International Conference on Physics beyond the Standard Model, Balholm, Norway, 1997. Eds. G. Eigen, P. Osland, B. Stugu. N. Y.: AIP, 1997. P. 354; hep-ph/9708423.

[5] V. A. Smirnov. Phys. Lett. B. 1997. V. 394. P. 205.

[6] A. Czarnecki, V. A. Smirnov. Phys. Lett. B. 1997. V. 394. P. 211.

[7] V. A. Smirnov. Phys. Lett. B. 1997. V. 404. P. 101.

[8] M. Beneke, V. A. Smirnov. Nucl. Phys. B. 1998. V. 522. P. 321.

[9] J. C. Collins. Sudakov form factors. In: Perturbative QCD. Ed. A. H. Mueller. Singapore: World Scientific, 1989. P. 573.

[10] В. В. Судаков. ЖЭЭФ. 1956. V. 30. Р. 87

[11] N. I. Ussyukina, A. I. Davydychev. Phys. Lett. B. 1993. V. 298. P. 363.

[12] G. 't Hooft, M. Veltman. Nucl. Phys. B. 1972. V. 44. P. 189; C. G. Bollini, J. J. Giambiagi. Nuovo Cimento B. 1972. V. 12. P. 20.

[13] R. J. Gonsalves. Phys. Rev. D. 1983. V. 28. P. 1542.

[14] K. G. Chetyrkin, F. V. Tkachov. Nucl. Phys. B. 1981. V. 192. P. 159.

[15] G. Kramer, B. Lampe. J. Math. Phys. 1997. V. 28. P. 945.

Поступила в редакцию 28.XII.1998 г. 